\title{
MÖGLICHST KONFORME SPIEGELUNG AN EINEM JORDANBOGEN AUF DER ZAHLENEBENE
}

\author{
Reiner Kühnau
}

\section{Einleitung}

Es sei $\mathfrak{c}$ ein abgeschlossener Jordanbogen mit Endpunkten $z_{1}$ und $z_{2}$ in der $z$-Ebene. Unter einer $Q$-quasikonformen Spiegelung an $\mathfrak{c}$ auf der Zahlenebene verstehen wir in dieser Mitteilung eine orientierungsumkehrende $Q$-quasikonforme Abbildung der $z$-Ebene auf sich mit Festhaltung des unendlich fernen Punktes (kurz $\infty \rightarrow \infty$ ), bei der $c$ punktweis festbleibt. In dieser Festhaltung des unendlich fernen Punktes liegt der Unterschied zur Mitteilung [5]. Obwohl im folgenden manche Überlegung in der Tendenz ähnlich wie in [5] ist, gibt es doch durch die Bedingung $\infty \rightarrow \infty$ einige wesentliche Unterschiede in der Durchführung und in den entstehenden Resultaten.

Eine quasikonforme Spiegelung an $\mathfrak{c}$ mit kleinstmöglicher Dilatationsschranke heißt (bei $\infty \rightarrow \infty$ ) "möglichst konform" im Anschluß an H. Grötzsch bzw. "extremal quasikonform" nach $\mathrm{O}$. Teichmüller. Die zugehörige kleinstmögliche Dilatationsschranke $\mathfrak{Q}_{\mathfrak{c}} \geq 1$ bzw. $\mathfrak{q}_{\mathfrak{c}}=\left(\mathfrak{Q}_{\mathfrak{c}}-1\right) /\left(\mathfrak{Q}_{\mathfrak{c}}+1\right)$ nennen wir "Spiegelungskoeffizient von $\mathfrak{c}$ (bei $\infty \rightarrow \infty$ )". Dieser ist invariant bei ganz-linearer Transformation der Ebene. Im Unterschiede hierzu war in [5] der ohne die Nebenbedingung $\infty \rightarrow \infty$ entstehende Spiegelungskoeffizient $Q_{\mathfrak{c}} \geq 1$ bzw. $q_{\mathfrak{c}}=\left(Q_{\mathfrak{c}}-1\right) /\left(Q_{\mathfrak{c}}+1\right)$ sogar invariant bei beliebiger linearer Transformation. Es gilt natürlich $\mathfrak{Q}_{\mathfrak{c}} \geq Q_{\mathfrak{c}}$ bzw. $\mathfrak{q}_{\mathfrak{c}} \geq q_{\mathfrak{c}}$. Wie bei $q_{\mathfrak{c}}$ gelten auch bei $\mathfrak{q}_{\mathfrak{c}}$ einfache naheliegende Monotonieeigenschaften $\left(\mathfrak{q}_{\mathfrak{c}}\right.$ vergrößert sich nicht bei Verkürzung von $\left.\mathfrak{c}\right)$.

Bildet man die zweiblättrige Riemannsche Fläche mit Windungspunkten bei $z_{1}$ und $z_{2}$ auf die schlichte $\mathfrak{z}$-Zahlenkugel so ab, daß die beiden Exemplare von $\infty$ in $\infty$ und 0 übergehen, entsteht aus $\mathfrak{c}$ eine geschlossene Jordankurve $\mathfrak{C}$. Aus einer $Q$-quasikonformen Spiegelung an c entsteht so eine $Q$-quasikonforme Spiegelung an $\mathfrak{C}$ mit der Nebenbedingung $\infty \rightarrow 0$; ebenso umgekehrt. Somit folgt $\mathfrak{q}_{\mathfrak{c}}=0$ genau für eine Strecke $\mathfrak{c}$ (nachdem zunächst in elementarer funktionentheoretischer Schlußweise aus $\mathfrak{q}_{\mathfrak{c}}=0$ folgt, daß $\mathfrak{C}$ ein Kreis ist). Die Bestimmung von $\mathfrak{q}_{\mathfrak{c}}$ für einen Kreisbogen $\mathfrak{c}$ ist gar nicht elementar (im Gegensatz zur Bestimmung von $q_{\mathfrak{c}}$ ), aber auf eine klassische Arbeit [9] von $\mathrm{O}$. Teichmüller zurückführbar; vgl. Sektion 2. Analog wie in [5] ergibt sich $\mathfrak{q}_{\mathfrak{c}}=|1-\gamma|$ für den Fall, $\mathfrak{c}$ besteht aus zwei unter dem Winkel $\gamma \pi$ zusammenstoßenden Strecken. Weitere Beispiele $\mathfrak{c}$ mit explizit angebbarem $\mathfrak{q}_{\mathfrak{c}}$ sind noch nicht bekannt. 
Durch die genannte Zurückführung auf die Spiegelung an der geschlossenen Jordankurve $\mathfrak{C}$ mit $\infty \rightarrow 0$ ergibt sich sofort aus Kompaktheitsgründen die Existenz mindestens einer möglichst konformen Spiegelung an $\mathfrak{c}$ mit $\infty \rightarrow \infty$. Daß diese für z.B. analytisches $c$ eindeutig bestimmt ist und sich durch ein quadratisches Differential (regulär bis auf eventuell einen einfachen $\mathrm{Pol}$ in $\infty$ ) charakterisieren läßt, folgt durch die Strebelsche Theorie möglichst konformer Abbildungen bei fest gegebenen Randwerten [8], [4].

Im folgenden soll nun die möglichst konforme Spiegelung an einem "kleinen" Bogen $\mathfrak{c}$ näher untersucht werden.

Satz 1. Es sei $\mathfrak{c}$ ein abgeschlossener Teilbogen eines festen analytischen Jordanbogens, wobei $\mathfrak{c}$ die Länge $2 \varepsilon$ besitzt und die Länge durch den festen Punkt $z_{0} \in \mathfrak{c}$ halbiert wird. Dann gilt für $\varepsilon \rightarrow 0$

$$
\mathfrak{q}_{\mathfrak{c}}=\frac{1}{4} k \varepsilon+O\left(\varepsilon^{2}\right)
$$

wobei $k$ die Krümmung in $z_{0}$ ist. $O\left(\varepsilon^{2}\right) / \varepsilon^{2}$ bleibt für $\varepsilon \rightarrow 0$ beschränkt.

Auch zur Geometrie der möglichst konformen Spiegelung an $\mathfrak{c}$ läßt sich für hinreichend kleines $\varepsilon$ unter der Voraussetzung $k \neq 0$ eine Aussage treffen.

Satz 2. Für alle hinreichend kleinen $\varepsilon$ ist das die möglichst konforme Spiegelung an $\mathfrak{c}$ (mit $\infty \rightarrow \infty$ ) beschreibende quadratische Differential bis auf einen einfachen Pol in $\infty$ außerhalb $\mathfrak{c}$ und auf den beiden Ufern von $\mathfrak{c}$ regulär und besitzt keine Nullstellen (in den Endpunkten von $\mathfrak{c}$ in einer Randuniformisierenden betrachtet).

Zur bequemeren Formulierung des folgendes Satzes (wieder unter der Voraussetzung kleiner $\varepsilon$ und $k \neq 0$ ), der diese Aussage noch weiter präzisiert, nehmen wir o.E.d.A. $\mathfrak{c}$ in der Lage von Figur 1 in der $z$-Ebene an $\left(z_{0}=0\right.$, horizontale Tangente in 0 , Vorzeichen der Krümmung so, daß $\mathfrak{c}$ oberhalb der reellen Achse liegt). Dann führen wir noch eine ähnliche Vergrößerung von $\mathfrak{c}$ durch eine ganzlineare Transformation in eine $\mathfrak{z}$-Ebene so aus, daß die Endpunkte von $\mathfrak{c}$ in \pm 2 übergehen und das Bild $\mathfrak{c}_{\mathfrak{z}}$ von $\mathfrak{c}$ unterhalb der reellen Achse liegt (Figur 1). Nun bilden wir die zweiblättrige Überlagerung des Äußeren von $\mathfrak{c}_{\mathfrak{z}}$ mit Windungspunkt in $\infty$ schlicht konform auf das Innere des Einheitskreises einer $\zeta$-Ebene so ab, daß $\mathfrak{z}=\infty$ in $\zeta=0$ übergeht und in $\mathfrak{z}=\infty$ die Entwicklung $a / \sqrt{i \mathfrak{z}}+\cdots(a>0)$ vorliegt. Als Schar $\mathfrak{S}$ bezeichnen wir dann in der $\mathfrak{z}$-Ebene die Gesamtheit der Urbilder der zur imaginären Achse parallelen Strecken innerhalb $|\zeta|<1$.

Satz 3. Bei der möglichst konformen Spiegelung an $\mathfrak{c}$ mit $\infty \rightarrow \infty$ gehen infinitesimale Kreise in infinitesimale Ellipsen des Achsenverhältnisses $\mathfrak{Q}_{\mathfrak{c}}$ über, wobei die großen Achsen einen Neigungswinkel besitzen, der in jedem Punkte der $\mathfrak{z}$-Ebene übereinstimmt mit dem Neigungswinkel der durch diesen Punkt verlaufenden Kurve von $\mathfrak{S}$ bis auf eine additive Fehlergröße, die nach Division durch $\sqrt{\varepsilon}$ beschränkt in $\mathfrak{z}$ (außerhalb von $\mathfrak{c}_{\mathfrak{z}}$ ) und $\varepsilon$ (hinreichend klein) ist. 

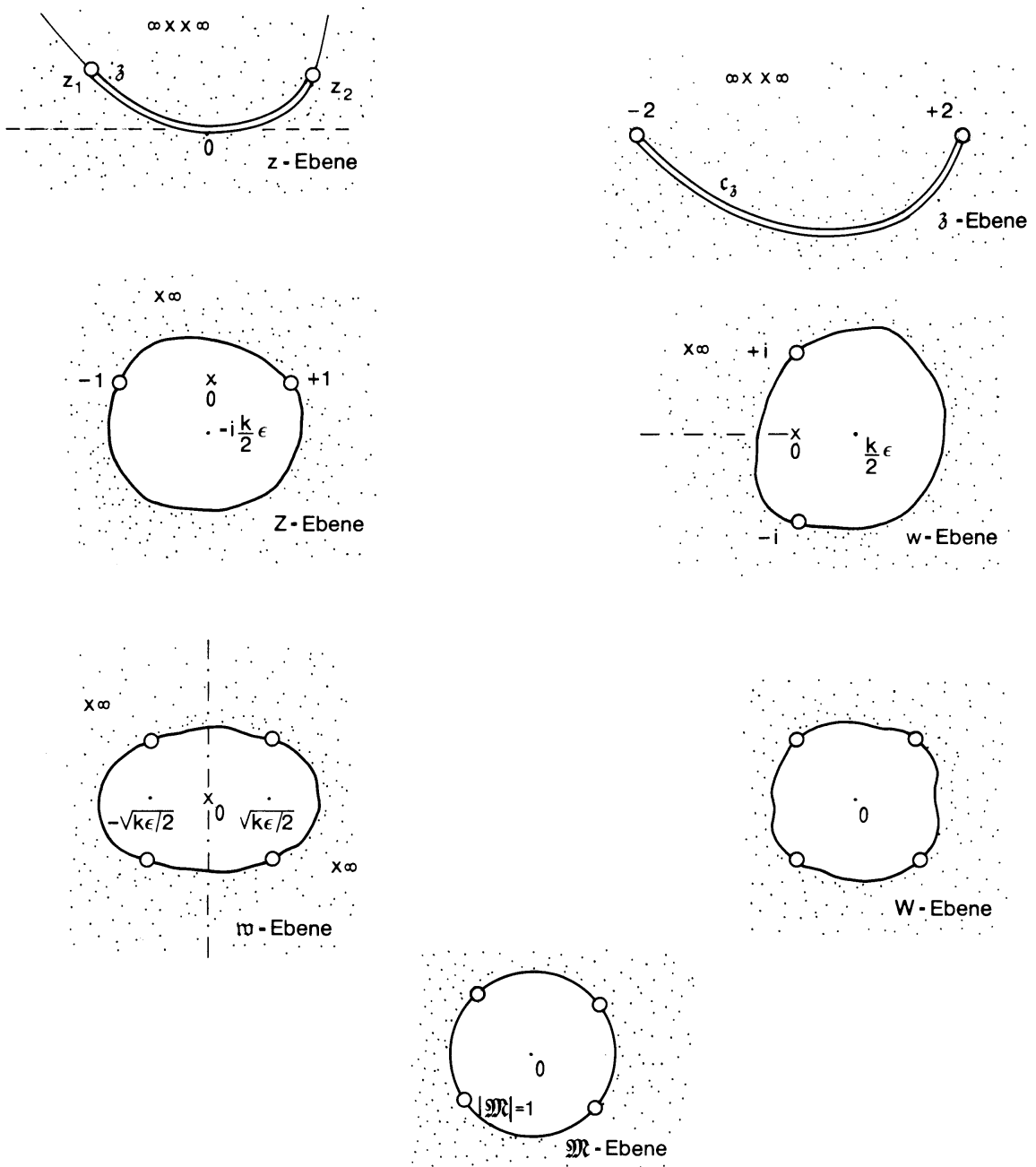

Figur 1.

Die Schar $\mathfrak{S}$ ist in Figur 2 dargestellt im Grenzfalle $\varepsilon=0$. Diese Schar entsteht dann also als Urbild der zur imaginären Achse parallelen Strecken innerhalb $|\zeta|<1$ bei schlichter konformer Abbildung des zweiblättrigen Äußeren (Windungspunkt in $\infty$ ) der Strecke $-2 \ldots+2$ der $\mathfrak{z}$-Ebene auf das Innere des Einheitskreises $|\zeta|<1$ mit $\infty \rightarrow 0$ und der Entwicklung $1 / \sqrt{i \mathfrak{z}}+\cdots$ in $\mathfrak{z}=\infty$. Die Schar $\mathfrak{S}$ ist (in diesem Grenzfalle $\varepsilon=0$ ) zur imaginären Achse symmetrisch. Bei Spiegelung an der reellen Achse entsteht die Schar der orthogonalen Trajektorien. Im oberen Ufer der Strecke $-2 \ldots+2$ endet die in $\mathfrak{z}$ einmündende Kurve von $\mathfrak{S}$ dort mit dem Neigungswinkel $\frac{1}{2}\left(\pi-\arcsin \frac{1}{2} \mathfrak{z}\right)$ gegen die positiv reelle Achse. 


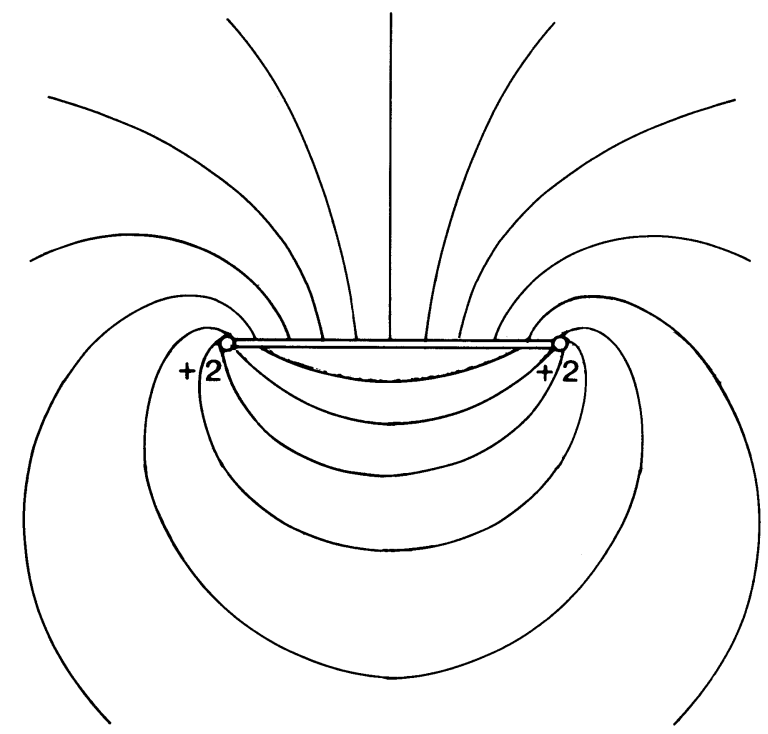

Figur 2. (Schar $\mathfrak{S}$ in der $\mathfrak{z}$-Ebene.)

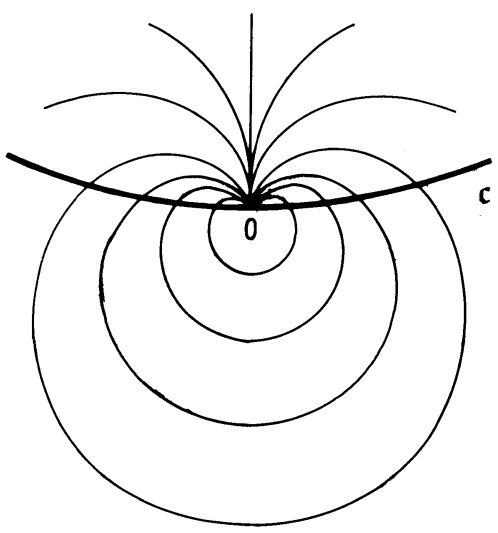

Figur 3. ( $z$-Ebene.)

Eine zu Satz 3 entsprechende Aussage ergibt sich für die $z$-Ebene, wobei dann die Neigungen der großen Achsen der infinitesimalen Bildellipsen analog außerhalb eines beliebig kleinen aber festen zu $z=0$ konzentrischen Kreises approximiert werden durch die Schar der Figur 3 (Cardioiden). (Diese Schar der Figur 3 entsteht aus derjenigen von Figur 2 sozusagen bei Betrachtung mit immer stärker werdender Verkleinerung.)

\section{Beispiel: Kreisbogen $c$}

Dieser Kreisbogen $\mathfrak{c}$ habe den Öffnungswinkel $\alpha(<2 \pi) . \mathfrak{q}_{\mathfrak{c}}$ hängt allein von $\alpha$ ab wegen der Invarianz bei Ähnlichkeitstransformation. $\mathbf{c}$ kann dabei mit den Endpunkten \pm 2 entsprechend der Situation in der $\mathfrak{z}$-Ebene der Figur 1 angenommen werden. Aus $\mathfrak{c}$ entsteht in der $Z$-Ebene der Figur 1 gemäß (11) eine exakte Kreislinie $K$, wobei man elementar für das Verhältnis $\varrho$ des Abstandes zwischen dem Mittelpunkt von $K$ und $Z=0$ zum Kreisradius errechnet

$$
\varrho=\sin (\alpha / 4) \text {. }
$$

Die Aufgabe der möglichst konformen Spiegelung an $K$ mit $\infty \rightarrow 0$ ist nun gerade in [9] vollständig behandelt worden. Es ergibt sich nach [9] (dort berühmter Schreibfehler auf S. 343: $K$ ist durch $\sqrt{K}$ zu ersetzen; gleicher Fehler in der Darstellung in [6], S. 59 ff.)

$$
\mathfrak{Q}_{\mathfrak{c}}=\left(e^{\mu(\varrho)}+1\right)^{2} /\left(e^{\mu(\varrho)}-1\right)^{2},
$$


wobei $\mu(\varrho)$ der Logarithmus des konformen Moduls der von 0 bis $\varrho$ geradlinig geschlitzten Einheitskreisscheibe ist. Mit der in [7, S. 62] gegebenen Darstellung für $\mu(\varrho)$ und der üblichen Bezeichnung für elliptische Integrale erster Gattung haben wir damit das Ergebnis:

$$
\mathfrak{q}_{\mathfrak{c}}=1 / \operatorname{Cos}\left(\frac{1}{2} \pi K^{\prime}(\varrho) / K(\varrho)\right)
$$

mit $\varrho$ nach (2).

Der topologische Verlauf der Hauptverzerrungsrichtungen der möglichst konformen Spiegelung am Kreisbogen $c$ ergibt sich nach [9] für alle $\alpha$ (also nicht nur für hinreichend kleine entsprechend Satz 3) wie in Figur 2.

Bemerkung. Man muß sich des Gedankens entschlagen, es finde immer eine Vergrößerung von $\mathfrak{q}_{\mathfrak{c}}$ statt, wenn man einen konvexen Bogen $\mathfrak{c}$ in Richtung der konvexen Seite abändert (bei Festhaltung der Endpunkte). Besteht nämlich $\mathfrak{c}$ aus zwei unter dem Winkel $\gamma \pi(<\pi)$ zusammenstoßenden gleich großen Strecken, ist nach der Einleitung $\mathfrak{q}_{\mathfrak{c}}=1-\gamma$. Für den Kreisbogen mit gleichen Endpunkten wie $\mathfrak{c}$ und durch den Knickpunkt verlaufend ergibt sich für kleine Werte von $1-\gamma$ nach (3) bzw. Satz 1 ein kleinerer Wert, nämlich asymptotisch $\pi(1-\gamma) / 4$.

\section{Beweis von Satz 1}

Dieser besteht im 1. Teil im Nachweis der Ungleichung

$$
\mathfrak{q}_{\mathfrak{c}} \geq \frac{1}{4} k \varepsilon+O\left(\varepsilon^{2}\right) .
$$

Dabei spielt eine zentrale Rolle eine Koeffizientenabschätzung für quasikonform fortsetzbare schlichte konforme Abbildungen. Im zweiten Beweisteile wird noch explizit eine Spiegelung konstruiert, für die der Betrag der komplexen Dilatation $\leq$ der rechten Seite von (4) ist.

Zum 1. Beweisteil vergleiche man Figur 1. Es wird eine Kette von schlichten konformen Abbildungen durchgeführt.

Den Bogen $\mathfrak{c}$ können wir o.E.d.A. in der $z$-Ebene in der Form

$$
z=x+i\left(\frac{1}{2} k x^{2}+O\left(x^{3}\right)\right), \quad k \geq 0,
$$

annehmen, wobei $k$ die Krümmung in $z_{0}=0$ ist,

$$
\begin{gathered}
z_{1}=\varepsilon+O\left(\varepsilon^{2}\right)+i\left(\frac{1}{2} k \varepsilon^{2}+O\left(\varepsilon^{3}\right)\right), \\
z_{2}=-\varepsilon+O\left(\varepsilon^{2}\right)+i\left(\frac{1}{2} k \varepsilon^{2}+O\left(\varepsilon^{3}\right)\right)
\end{gathered}
$$

die Endpunkte sind. Es wird

$$
z_{1}-z_{2}=2 \varepsilon+2 \varepsilon O_{1}(\varepsilon)+i O\left(\varepsilon^{3}\right),
$$




$$
z_{1}+z_{2}=2 \varepsilon O_{2}(\varepsilon)+2 i\left(\frac{1}{2} k \varepsilon^{2}+O\left(\varepsilon^{3}\right)\right) .
$$

Hierbei und im folgenden bezeichnet $O$ das Landau-Symbol und zwar stets eine reelle Funktion. Wir hängen an $O$ einen Index an, wenn wir eine ganz bestimmte Funktion meinen.

a). Übergang $z \rightarrow \mathfrak{z}$ als erstes Glied der angekündigten Abbildungskette: Erfolgt durch die ganz-lineare Transformation

$$
\mathfrak{z}=\frac{4}{z_{1}-z_{2}} z-2 \frac{z_{1}+z_{2}}{z_{1}-z_{2}}
$$

so daß der Bildbogen in der $\mathfrak{z}$-Ebene die Endpunkte \pm 2 bekommt. Der Bildbogen hat nach (5) die Gestalt

$$
\mathfrak{z}=2 \frac{x+i\left(\frac{1}{2} k x^{2}+O\left(x^{3}\right)\right)-\varepsilon O_{2}(\varepsilon)-i\left(\frac{1}{2} k \varepsilon^{2}+O\left(\varepsilon^{3}\right)\right)}{\varepsilon+\varepsilon O_{1}(\varepsilon)+i O\left(\varepsilon^{3}\right)},
$$

wieder mit $x$ als Kurvenparameter. Wir steigen nun zum Kurvenparameter $\varphi$ durch

$$
x=\left(\varepsilon+\varepsilon O_{1}(\varepsilon)\right) \cos \varphi+\varepsilon O_{2}(\varepsilon), \quad 0 \leq \varphi \leq \pi,
$$

um. Die Endpunkte des Bildbogens bekommen wir jetzt durch $\varphi=0$ und $\varphi=\pi$, wobei dort von $x$ der Wert $\mathfrak{R e} x_{1}$ bzw. $\mathfrak{R e} x_{2}$ von zweiter Ordnung angenommen wird. Wir stellen uns noch vor, für $\pi \leq \varphi \leq 2 \pi$ entsteht das andere Ufer des Schlitzes bei $\mathfrak{c}$.

Nun wird in diesem neuen Parameter $\varphi$ nach (7) der Bildbogen in der $\mathbf{z}$-Ebene gegeben durch

$$
\mathfrak{z} / 2=\frac{\left(1+\frac{1}{2} i k \varepsilon \cos \varphi\right) \cos \varphi+O_{1}(\varepsilon) \cos \varphi+i O\left(\varepsilon^{2}\right)-\frac{1}{2} i k \varepsilon}{1+O_{1}(\varepsilon)+i O\left(\varepsilon^{2}\right)},
$$

wobei von jetzt ab das Landau-Symbol $O$ (ohne Index) auch zusätzlich von $\varphi$ abhängen kann, dabei immer als analytisch in $\varepsilon$ und $\varphi$ vorzustellen ist. Es ist $O\left(\varepsilon^{2}\right) / \varepsilon^{2}$ für $\varepsilon \rightarrow 0$ beschränkt, und zwar gleichmäßig in $\varphi$ (Entsprechendes gilt unten).

Wir rechnen noch um zu

$$
\mathfrak{z} / 2=\cos \varphi-\frac{1}{2} i k \varepsilon \sin ^{2} \varphi+O\left(\varepsilon^{3}\right)+i O\left(\varepsilon^{2}\right) .
$$

Hier muß $O\left(\varepsilon^{3}\right)+i O\left(\varepsilon^{2}\right)$ für $\varphi=0$ und $\varphi=\pi$ von zweiter Ordnung verschwinden, so daß also diese Größe nach Division durch $\varepsilon^{2} \sin ^{2} \varphi$ beschränkt sein muß. Dies bringt

$$
(\mathfrak{z} / 2)^{2}-1=-\sin ^{2} \varphi\left(1+i k \varepsilon \cos \varphi+O\left(\varepsilon^{2}\right)+i O\left(\varepsilon^{2}\right)\right),
$$




$$
\left((z / 2)^{2}-1\right)^{1 / 2}=i \sin \varphi\left(1+\frac{1}{2} i k \varepsilon \cos \varphi+O\left(\varepsilon^{2}\right)+i O\left(\varepsilon^{2}\right)\right) .
$$

b). Übergang $z \rightarrow Z:$ Erfolgt durch

$$
\mathfrak{z}=Z+1 / Z, \quad Z=(\mathfrak{z} / 2)+\sqrt{(\mathfrak{z} / 2)^{2}-1} .
$$

Mit (9), (10) wird die Bildkurve (eine geschlossene analytische Jordankurve) in der $Z$-Ebene dargestellt durch

$$
Z=e^{i \varphi}\left(1-\frac{1}{2} k \varepsilon \sin \varphi\right)+O\left(\varepsilon^{2}\right)+i O\left(\varepsilon^{2}\right) .
$$

Durch die weitere Substitution $\varphi=\psi-\frac{1}{2} k \varepsilon \cos \psi$ entsteht daraus die Darstellung

$$
Z=e^{i \psi}-\frac{1}{2} i k \varepsilon+O\left(\varepsilon^{2}\right)+i O\left(\varepsilon^{2}\right) .
$$

c). Übergang $Z \rightarrow w$ : Ist eine harmlose Drehung

$$
w=i Z \text {. }
$$

Aus (12) entsteht die Bildkurve

$$
w=i e^{i \psi}+\frac{1}{2} k \varepsilon+O\left(\varepsilon^{2}\right)+i O\left(\varepsilon^{2}\right) .
$$

d). Übergang $w \rightarrow \mathfrak{w}:$ Durch

$$
\mathfrak{w}=\sqrt{w} .
$$

In der $\mathfrak{w}$-Ebene entsteht dann die Bildkurve in der Form

$$
\mathfrak{w}=i e^{i(2 \psi+\pi) / 4}+\frac{1}{4} k \varepsilon^{-i(2 \psi+\pi) / 4}+O\left(\varepsilon^{2}\right)+i O\left(\varepsilon^{2}\right) .
$$

e). Übergang $\mathfrak{w} \rightarrow W:$ Erfolgt durch

$$
\mathfrak{w}=W+\frac{1}{4} k \varepsilon \frac{1}{W}, \quad W=\mathfrak{w}-\frac{1}{4} k \varepsilon \frac{1}{\mathfrak{w}}+\frac{1}{\mathfrak{w}^{3}}+\cdots .
$$

Die Bildkurve in der $W$-Ebene ist dann gegeben durch

$$
W=i e^{i(2 \psi+\pi) / 4}+O\left(\varepsilon^{2}\right)+i O\left(\varepsilon^{2}\right) .
$$

Wegen

$$
|W|=1+O\left(\varepsilon^{2}\right)
$$


haben wir eine Annäherung an den Einheitskreis von zweiter Ordnung. Das Äußere dieser Bildkurve hat einen konformen Radius der Form

$$
R=1+O\left(\varepsilon^{2}\right) .
$$

f). Übergang $W \rightarrow \mathfrak{W}$ : Erfolgt durch schlichte konforme Abbildung des Äußeren der Kurve der $W$-Ebene auf das Äußere des Einheitskreises, wobei für die Umkehrfunktion in $\mathfrak{W}=\infty$ der Entwicklungstypus (wegen der zentrischen Symmetrie zu 0)

$$
W / R=\mathfrak{W}+\mathfrak{A}_{1} \mathfrak{W}^{-1}+\mathfrak{A}_{3} \mathfrak{W}^{-3}+\cdots
$$

vorliegt. Entsprechend wie in [5] fließt aus (19)

$$
\left|\mathfrak{A}_{1}\right| \leq O\left(\varepsilon^{2}\right) .
$$

Nun haben wir nach (17) in $\mathfrak{W}=\infty$ die Entwicklung

$$
\frac{\mathfrak{w}}{R}=\frac{W}{R}+\frac{1}{4} k \varepsilon R^{-2} \frac{R}{W}=\mathfrak{W}+\left(\mathfrak{A}_{1}+\frac{1}{4} k \varepsilon R^{-2}\right) \mathfrak{W}^{-1}+\mathfrak{W}^{-3} .
$$

Aus der $\mathfrak{Q}_{\mathfrak{c}}$-quasikonformen Spiegelung an $\mathfrak{c}$ mit $\infty \rightarrow \infty$ entsteht entsprechend der Einleitung eine $\mathfrak{Q}_{\mathfrak{c}}$-quasikonforme Spiegelung an der "ellipsennahen" Kurve der $\mathfrak{w}$-Ebene mit $\infty \rightarrow 0$, und diese induziert in bekannter Weise (vgl. z.B. [4]) eine $\mathfrak{Q}_{\mathfrak{c}}$-quasikonforme Fortsetzung der schlichten hydrodynamisch normierten konformen Abbildung (23) des Äußeren des Einheitskreises der $\mathfrak{W}$-Ebene auf das $\ddot{A} u ß e r e$ der Kurve der Ebene $\mathfrak{w} / R$.

Nun kommt der entscheidende Schritt in diesem ersten Beweisteil: Nach [1] können wir so in (23) den ersten Koeffizienten abschätzen

$$
\left|\mathfrak{A}_{1}+\frac{1}{4} k \varepsilon R^{-2}\right| \leq \mathfrak{q}_{\mathfrak{c}} .
$$

Das gibt im Verein mit (20), (22) unsere Behauptung (4).

Im 2. Beweisteil zu Satz 1 wird effektiv an der zu 0 zentrisch symmetrischen geschlossenen Jordankurve der $\mathfrak{w}$-Ebene (vgl. wieder Figur 1) eine quasikonforme Spiegelung konstruiert, deren Betrag der komplexen Dilatation $\leq \frac{1}{4} k \varepsilon+O\left(\varepsilon^{2}\right)$ ist und die ebenfalls zu 0 zentrisch symmetrisch ist (so daß sie - wenn man in Figur 1 die verschiedenen Ebenen zurückverfolgt - zu einer quasikonformen Spiegelung an $\mathfrak{c}$ mit $\infty \rightarrow \infty$ Anlaß gibt, bei gleicher Dilatationsschranke). Diese Spiegelung wird analog und fast wörtlich wie in [5] (dort 2. Beweisteil in Sektion 3) konstruiert. Im wesentlichen ist nur $A$ durch $\frac{1}{2} k$ zu ersetzen, und in den Potenzen von $\varepsilon$ ist der Exponent entsprechend zu erniedrigen. 


\section{Beweis von Satz 2 und Satz 3}

Zum näheren Studium der möglichst konformen Spiegelung an $\mathfrak{c}$ mit $\infty \rightarrow$ $\infty$ untersuchen wir wie in [5] die (zentrisch symmetrische) möglichst konforme Spiegelung an der geschlossenen Jordankurve der $\mathfrak{w}$-Ebene oder - was hierzu nach [4] äquivalent ist - die möglichst konforme Fortsetzung der schlichten konformen Abbildung $\mathfrak{w}(\mathfrak{W})$ von $|\mathfrak{W}|>1$. Letztere Fortsetzung erhalten wir wie im Beweis von Satz 7 in [2] (dort Druckfehler: lies $F(w(1 / \bar{z}))$ ), [3](Satz 1) aus der komplexen Eigenfunktion zur Jordankurve der $\mathfrak{w}$-Ebene, aufgefaßt als Funktion von $\mathfrak{W}$,

$$
F(\mathfrak{W})=i \sum_{n=1}^{\infty} \overline{x_{n}} \mathfrak{W}^{-n} / \sqrt{n}, \quad(|\mathfrak{W}|>1), \quad \sum\left|x_{n}\right|^{2}=1
$$

falls diese schlicht ist.

Für hinreichend kleine $\varepsilon$ gilt für den Koeffizienten $a_{1}$ von $1 / \mathfrak{W}$ in (23)

$$
a_{1}=\frac{1}{4} k \varepsilon+O\left(\varepsilon^{2}\right), \quad\left|a_{1}\right|^{2}=\frac{1}{16} k^{2} \varepsilon^{2}+O\left(\varepsilon^{3}\right) .
$$

Andererseits ist $\mathfrak{w}(\mathfrak{W})$ nach $|\mathfrak{W}|>\varrho$ schlicht und analytisch fortsetzbar, wobei wählbar ist

$$
\varrho=\operatorname{const} \sqrt{\varepsilon}
$$

mit einer von $\varepsilon$ unabhängigen Konstanten. Denn an dem Bogen der $\mathfrak{z}$-Ebene zwischen -2 und +2 läßt sich in einem Umgebungsstreifen einer Breite der Form const $(1 / \varepsilon)$ spiegeln, und diese Spiegelung gibt in bekannter Weise Anlaß zu einer schlichten und analytischen Fortsetzung von $\mathfrak{w}(\mathfrak{W})$ nach $|\mathfrak{W}|>\operatorname{const} \sqrt{\varepsilon}$. Die Schlichtheit der Eigenfunktion (25) und Satz 2 ergeben sich nun aus (26), (27) nach Satz 5 in [5], dort insbesondere Bemerkungen 2 und 3 (hier wird $\Phi(\varrho)=$ const $\varepsilon^{3}+$ $\left.O\left(\varepsilon^{7 / 3}\right)\right)$. Auch folgt noch nach Satz 5 in [5] für den reziproken Fredholmschen Eigenwert $\kappa$ der Jordankurve der $w$-Ebene

$$
\kappa=\mathfrak{q}_{\mathfrak{c}}=\frac{1}{4} k \varepsilon+O\left(\varepsilon^{2}\right) .
$$

Nun zum Beweis von Satz 3! Es gilt zunächst (vgl. die Analogie zu (40) in

$$
\sum_{n=2}^{\infty} \sqrt{n}\left|x_{n}\right||\mathfrak{W}|^{-n-1} \leq c \sqrt{\varepsilon}\left|x_{1}\right||\mathfrak{W}|^{-2} \quad \text { für }|\mathfrak{W}| \geq 1
$$

mit einer gewissen Konstanten $c$ und für hinreichend kleine $\varepsilon$. Denn (29) folgt aus

$$
\sum_{n=2}^{\infty} \sqrt{n}\left|x_{n}\right| \leq c \sqrt{\varepsilon}\left|x_{1}\right|
$$


und dies wie in [5] aus

$$
\left(\sum_{n=2}^{\infty} \sqrt{n} \varrho^{n+1}\right)^{2}+c^{2} \varepsilon \frac{\varrho^{6}}{1-\varrho^{2}} \leq c^{2} \varepsilon \kappa^{2},
$$

was für kleine $\varepsilon$ sicher mit einer geeigneten Konstanten $c$ richtig ist wegen (27), (28). Es gilt weiter wie in [5]

$$
\left|\kappa x_{1}-\overline{a_{1} x_{1}}\right| \leq \frac{1}{\kappa} \frac{\varrho^{6}}{1-\varrho^{2}} .
$$

Mit (26) und (28) (hier benutzen wir also Satz 2) ergibt sich hieraus

$$
\mathfrak{I m} x_{1}=O(\varepsilon) .
$$

Ferner gilt

$$
\sum_{n=2}^{\infty}\left|x_{n}\right|^{2} \leq \frac{1}{\kappa^{2}} \frac{\varrho^{6}}{1-\varrho^{2}}=O(\varepsilon), \quad\left|x_{1}\right|^{2}=1-\sum_{n=2}^{\infty}\left|x_{n}\right|^{2}=1-O(\varepsilon),
$$

so daß folgt

$$
x_{1}=1-O(\varepsilon)+i O(\varepsilon) .
$$

Damit gilt für die Ableitung der komplexen Eigenfunktion (25) nach (29)

$$
\frac{d}{d \mathfrak{W}} F(\mathfrak{W})=-i \mathfrak{W}^{-2} \overline{x_{1}}\left(1+c \sqrt{\varepsilon} F^{*}(\mathfrak{W}, \varepsilon)\right) \quad \text { für }|\mathfrak{W}| \geq 1
$$

mit $\left|F^{*}(\mathfrak{W}, \varepsilon)\right| \leq 1$ für $|\mathfrak{W}| \geq 1$ und alle hinreichend kleinen $\varepsilon$. Das beweist Satz 3, da die Schar $\mathfrak{S}$ - in die $\mathfrak{W}$-Ebene überpflanzt - durch $\mathfrak{I m}_{\mathfrak{m}} i \mathfrak{W}^{-1}=$ const charakterisiert wird, die eigentliche Schar der Hauptverzerrungsrichtungen (Richtungen der großen Achsen der infinitesimalen Ellipsen, die Bilder infinitesimaler Kreise) durch $\mathfrak{I m} d F=0$.

\section{Literatur}

[1] KÜHNAU, R.: Wertannahmeprobleme bei quasikonformen Abbildungen mit ortsabhängiger Dilatationsbeschränkung. - Math. Nachr. 40, 1969, 1-11.

[2] KühNAU, R.: Quasikonforme Fortsetzbarkeit, Fredholmsche Eigenwerte und Grunskysche Koeffizientenbedingungen. - Ann. Acad. Sci. Fenn. Ser. A I Math. 7, 1982, 383-391.

[3] KüHNAU, R.: Wann sind die Grunskyschen Koeffizientenbedingungen hinreichend für $Q$ quasikonforme Fortsetzbarkeit? - Comment. Math. Helv. 61, 1986, 290-307.

[4] KühnaU, R.: Möglichst konforme Spiegelung an einer Jordankurve. - Jahresber. Deutsche Math. Ver. 90, 1988, 90-109. 
[5] KühNAU, R.: Möglichst konforme Spiegelung an einem Jordanbogen auf der Zahlenkugel. - Pfluger Anniversary Volume, Birkhäuser Verlag, Basel-Boston-Berlin, 1988, 139156.

[6] KüNZI, H.P.: Quasikonforme Abbildungen. - Springer Verlag, Berlin-Göttingen-Heidelberg, 1960 .

[7] Lehto, O., and K.I. Virtanen: Quasikonforme Abbildungen. - Springer Verlag, BerlinHeidelberg-New York, 1965.

[8] Strebel, K.: On the existence of extremal Teichmueller mappings. - J. Analyse Math. $30,1976,464-480$.

[9] Teichmüller, O.: Ein Verschiebungssatz der quasikonformen Abbildung. - Deutsche Math. 7, 1944, 336-343. Auch in: Gesammelte Abhandlungen, Springer-Verlag, Berlin-Heidelberg-New York, 1982.

Martin-Luther-Universität Halle-Wittenberg

Sektion Mathematik

DDR-40 Halle an der Saale

Received 27 October 1988 